\title{
Ice Nucleation Properties of Soil Derived Mineral and Soil Organic Particles
}

\author{
Swarup China ${ }^{1 *}$, Gourihar Kulkarni ${ }^{1}$, Kuo-Pin Tseng ${ }^{1}$ and Noopur Sharma ${ }^{1}$ \\ 1. Pacific Northwest National Laboratory, Richland, WA, USA. \\ * Corresponding author: swarup.china@pnnl.gov
}

Soil organic and mineral particles are emitted to the atmosphere through recently discovered landatmosphere interactions (e.g., raindrop impaction on soil surface) [1-3]. However, there is, an ongoing debate on whether these aerosols can influence the background atmospheric aerosol budget and further, whether they influence cold cloud formation by acting as ice nucleating particles (INP). Heterogeneous ice nucleation processes and the concentration of INPs influence the formation of ice cloud, thus affecting cloud radiative properties and precipitation. These processes depend upon the physical and chemical properties of INPs, and presently such properties are poorly characterized.

In this study we investigate natural soils samples for predictive assessment of the emission of soil organic particles, via aerosolization of particles upon impingement of rain droplets on soil surface. Soil organic and mineral particles are generated through simulated raindrop impact on the soil surface. Preliminary results show significant amount of particles are generated upon impingement of rain droplets on soil surface (Figure 1). We also performed ice nucleation experiments to understand the role of these particles on their ice nucleation efficiency. Ice nucleation experiments are performed using a continuous flow diffusion chamber, simulating immersion freezing under mixed-phase cloud conditions and using a custom-built ice nucleation cryo-stage, interfaced with an environmental scanning electron microscope operated at temperature and relative humidity relevant for cirrus cloud formation.

Figure 2 shows an example of dynamic observation of ice nucleation events on individual mineral particle. Physicochemical properties of individual ice nucleating particles and particle populations are characterized using multimodal micro-spectroscopy and chemical imaging techniques. We will present ice nucleation efficiency of both mixed mineral and organic components of soil particles. These results may have implications towards predicting ice nucleation in cloud model.

\section{References:}

[1] YS Joung, CR Buie, Nature Communications 6 (2015), p. 6083.

[2] YS Joung, Z Ge and CR Buie, Nature Communications 8 (2017), p. 14668.

[3] B Wang et al., Nature Geoscience 9 (6) (2016), p. 433. 


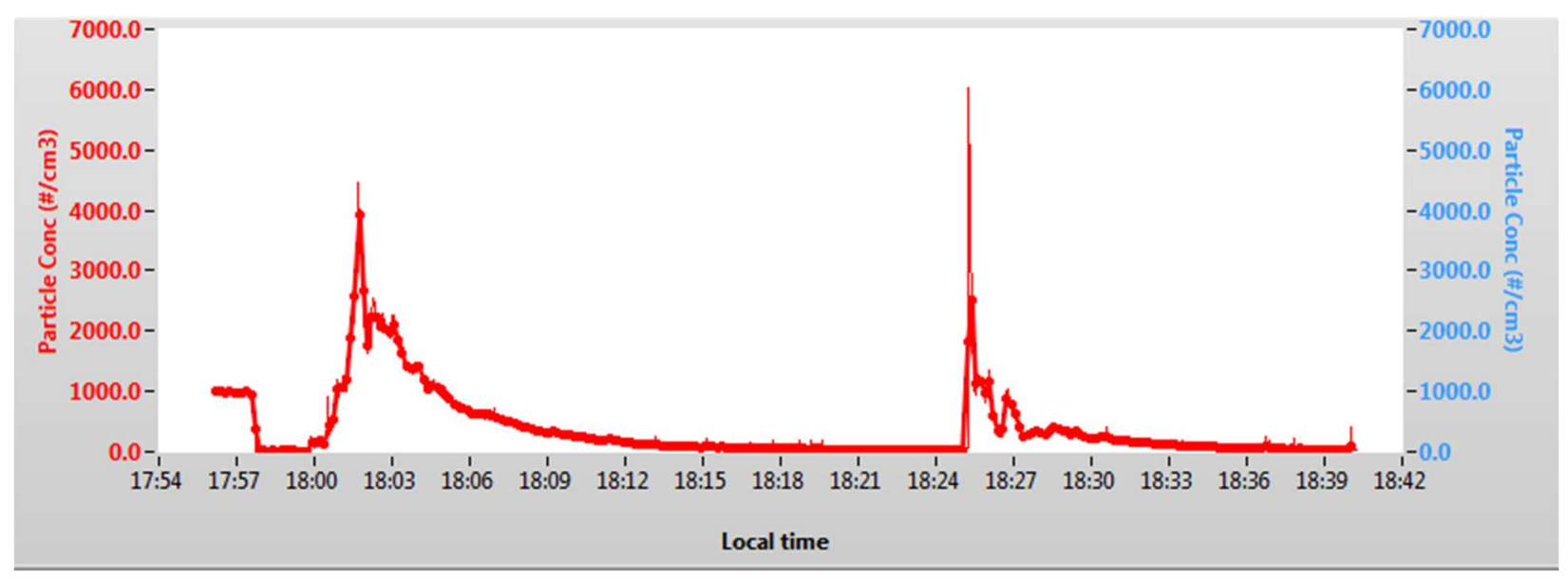

Figure 1. Concentration of particles emitted after one raindrop size droplet impacted the soil surface. Second droplet was released after $\sim 25$ minutes, but fewer particles were emitted.
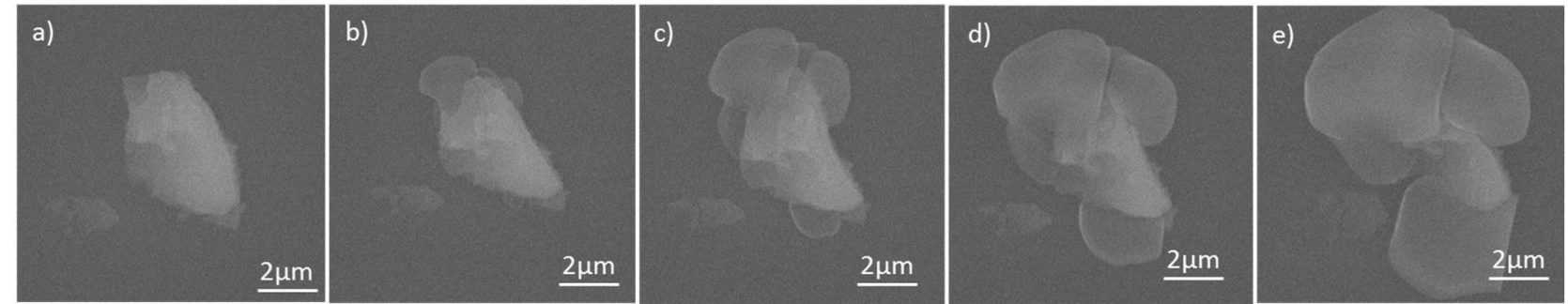

Figure 2. Dynamic observation of ice nucleation events on individual mineral particle. a) Before ice formation, b) initiation of ice formation, and c-e) subsequent growth of ice crystal. 